\title{
JOINT DIRECTION-OF-ARRIVAL AND ARRAY SHAPE TRACKING FOR MULTIPLE MOVING TARGETS
}

\author{
Jason Goldberg \\ Department of Electrical Engineering-Systems \\ Tel Aviv University, Tel Aviv 69978, Israel \\ jasondeng.tau.ac.il
}

\author{
Ana Pérez-Neira and Miguel Angel Lagunas \\ Departament de Teoria del Senyal i Comunicacions \\ E.T.S.E.T. Campus Nord, UPC. \\ Mod. D-5, Gran Capità. s/n, 08034 Barcelona, Spain. \\ anuska@gps.tsc.upc.es
}

\begin{abstract}
An algorithm for the joint tracking of source DOA's and sensor positions is presented to address the problem of DOA tracking in the presence sensor motion. Initial maximum likelihood estimates of source DOA's and sensor positions are refined by Kalman filtering. Spatio-temporally correlated array movement is considered. Source angle dynamics are used to achieve correct data association. The new technique is capable of performing well for the difficult cases of sources that cross in angle, fully coherent sources, as well as sources of identical or vastly different (possibly time-varying) power. Computer simulations show that the approach is robust in the presence of array motion modeling uncertainty and effectively reduces dependence on expensive and possibly unreliable hardware.
\end{abstract}

\section{INTRODUCTION}

The issue of tracking the directions-of-arrival (DOA's) of moving sources or targets has begun to receive increased attention, e.g., [1]. The "DOA tracking problem" consists of estimating the DOA's of moving sources in such a way that each of the DOA estimates is correctly associated with a particular source from one increment of time to the next. In the specific case of passive towed arrays for sonar, the accuracy of source tracking algorithms can be greatly limited by sensor location uncertainty [2]. This uncertainty may be due to the motion of the towing vessel, ocean conditions, as well as the physical properties of the array.

To deal with this problem, two general approaches to array shape estimation have emerged over the past several years. The first is based on the application of "interpolation type" algorithms applied to the outputs of depth and orientation sensors distributed along the array, e.g., [3]. Also, specifically for array shape tracking, [4], [5] makes use of the Paidoussis equation as a dynamic wave propagation model for a thin flexible underwater line array subject to motion induced at the tow point. Secondly, to avoid the excessive cost and practical difficulties of using a high number of depth sensors and compasses, several socalled data-driven "self-calibration" techniques have been proposed e.g., [6]. In the absence of other modeling errors, data driven techniques offer the possibility of accuracy limited only by the number of snapshots available [2]. However, such methods treat the sensor displacements as spatio-temporally uncorrelated random variables of known statistics or as deterministic unknowns.

This work has been supported by EEC Contract HCM/CHRXCT-930405, National Plan of Spain CICYT, Grant Number TIC-96-0500-C10-01, CIRIT 1996 SGR0096, and the Fulbright Program. J. Goldberg was with UPC.
This paper seeks to combine the advantages of the two basic approaches described above in order to solve the problem of jointly tracking the DOA's of multiple moving targets and the array shape. The main contribution of the paper is the combination of data driven maximum likelihood estimates (MLE's) followed by Kalman filtering which allows one to gain robustness in the presence of sensor motion modeling uncertainties while effectively minimizing the tracker's dependence on potentially costly and unreliable depth sensor and/or compass hardware.

\section{PROBLEM FORMULATION}

\subsection{Preliminary Notation}

First, consider $N$ spatially stationary far-field point sources impinging on a stationary array of $M$ passive sensors in the presence of additive white Gaussian noise (AWGN) over an observation interval of duration $T_{o}$. The output of the $m$ th sensor can be written as [7]:

$$
z_{m}(t)=\sum_{n=1}^{N} s_{n}\left(t-\tau_{m n}\right)+e_{m}(t), \quad t \in\left[0, T_{o}\right)
$$

where $\left\{s_{n}(t)\right\}_{n=1}^{N}$ and $\left\{e_{m}(t)\right\}_{m=1}^{M}$ denote the signal and sensor noise waveforms, respectively, and $\left\{\tau_{m n}\right\}$ are the propagation delays from the $n$th source to the $m$ th sensor. Divide the temporal interval into $B$ sub-intervals. The frequency domain snapshot vector at frequency $\omega$ and $b$ th temporal sub-interval is written as:

$$
\mathbf{z}(b, \omega)=\mathbf{A}(\omega) \mathbf{s}(b, \omega)+\mathbf{e}(b, \omega), b \in\{1, \cdots, B\}
$$

where $\mathbf{A}(\omega)$ is the $M \times N$ steering matrix at frequency $\omega$, and $\mathbf{s}(b, \omega)$ and $\mathbf{e}(b, \omega)$ are, respectively, the $N$ and $M$ dimensional signal and noise Fourier coefficient vectors at frequency $\omega$ and sub-interval $b$.

Restricting attention to far-field, point sources at frequency $\omega$ and co-planar with the array we can write:

$$
\begin{aligned}
{[\mathbf{A}(\omega)]_{m n} } & =e^{-j \omega \tau_{m n}\left(\theta_{n}\right)}, \\
\tau_{m n}\left(\theta_{n}\right) & =-\frac{1}{c}\left(x_{m} \cos \theta_{n}+y_{m} \sin \theta_{n}\right)
\end{aligned}
$$

where $\left\{\left(x_{m}, y_{m}\right)\right\}_{m=1}^{M}$ denote the positions in the $x y$ plane of the $M$ sensors. $\left\{\theta_{n}\right\}_{n=1}^{N}$ denote the DOA's of the $N$ sources. The notation $[\cdot]_{m n}$ denotes the $m n$th element of a matrix.

2.2. DOA and Array Shape Dynamical Model

Now, consider the spatially nonstationary case of source DOA's and array shape that change with time. Begin by 
assuming that each of the $N$ DOA trajectories as a function of time is described by the following discrete time state space equation with update interval $T$ :

$$
\begin{aligned}
\mathbf{x}_{\theta_{n}}(k+1) & =\mathbf{F}_{\theta} \mathbf{x}_{\theta_{n}}(k)+\mathbf{w}_{\theta_{n}}(k) \\
\mathbf{x}_{\theta_{n}}(k) & =\left[\theta_{n}(k) \dot{\theta}_{n}(k)\right]^{T}, \quad \mathbf{F}_{\theta}=\left[\begin{array}{ll}
1 & T \\
0 & 1
\end{array}\right]
\end{aligned}
$$

where the angle update time index $k$ corresponds to time $t=k T . \quad \theta_{n}(k)$ and $\dot{\theta}_{n}(k)$ respectively denote the angular position and speed of the $n$th source at time $k$. The model noise, $\mathrm{w}_{\theta_{n}}(k)$, is assumed zero mean, Gaussian and of covariance $\mathbf{Q}_{\theta_{n}}(k)$. (5) describes a so-called "constant velocity" model for the DOA trajectories subject to random perturbations. This model is accurate if the source motion is sufficiently slow relative to the parameter update interval, $T$.

Next, consider a model for the dynamic behavior of the array shape. For simplicity, consider the uniform linear array (ULA) with unknown sensor movement restricted to the $y$-axis, (i.e., the $x$-coordinates of each of the sensor locations is assumed known). A sensor movement model incorporating spatially as well as temporally correlated type motion is that of the Paidoussis equation e.g., [4].

A discretized state space formulation of the simplified "water pulley" version of the Paidoussis equation in addition to depth and/or compass sensor measurements were used as the foundation of a Kalman based array shape tracker in [4]. The model is also used in the present work. As in other data driven techniques, it will be assumed in the model that the positions of sensors one and two are known. ${ }^{1}$ The dynamic model will then applied to describe the motion for sensors three to $M$ :

$$
\begin{aligned}
\mathbf{x}_{\mathbf{y}}(k+1) & =\mathbf{F}_{\mathbf{y}} \mathbf{x}_{\mathbf{y}}(k)+\mathbf{w}_{\mathbf{y}}(k)+\mathbf{u}(k), \\
\mathbf{x}_{\mathbf{y}}(k) & =\left[y_{\mathbf{3}}(k), \cdots, y_{M}(k)\right]^{T}, \\
\mathbf{u}(k) & =\left[\rho y_{2}(k-1), 0, \cdots, 0\right]^{T}, \\
\mathbf{Q}_{\mathbf{y}}(k) & =E\left[\mathbf{w}_{\mathbf{y}}(k) \mathbf{w}_{\mathbf{y}}^{\mathrm{T}}(k)\right] \\
\mathbf{F}_{\mathbf{y}} & =(1-\rho) \mathbf{I}+\rho \mathbf{L}, \quad \mathbf{L}=\left[\begin{array}{cccc}
0 & 0 & \cdots & 0 \\
1 & 0 & & \vdots \\
0 & 1 & 0 & \\
\vdots & & \ddots & \\
0 & \cdots & & 1
\end{array}\right]
\end{aligned}
$$

where $\mathbf{u}(k)$ is a driving term which is assumed known in this work. $\rho \in[0,1]$ is a parameter, which, for water pulley motion, is the ratio of the distance traveled by the disturbance along the array in $T$ seconds to the spatial discretization interval (corresponding to the distance between adjacent sensors in our case). (6) models the position of each sensor $m \in\{3, \cdots, M\}$ as a weighted sum of its position at the previous time instant and that of the previous adjacent sensor at the previous time instant plus zero mean additive white Gaussian noise (AWGN) of specified variance.

As explained in [4], if $T$ is chosen such that $\rho=1$, then $\boldsymbol{F}_{\mathbf{y}}$ has been found to be well approximated by $\alpha \mathbf{L}$, where

\footnotetext{
${ }^{1}$ Again, in practice, estimates of these positions will be obtained from some type of additional depth sensor and/or compass hardware.
}

$\alpha$ is a damping factor near unity. Also, it is seen that for $\rho=0$, the sensor motion is independent from one sensor to the next. In this case (6) corresponds to a first order auto-regressive (AR) model to describe the displacement of each sensor from the array axis as a function of time. In this case, the position of each sensor is modeled as its position at the previous time instant plus AWGN.

\subsection{DOA/Array Shape Tracking Problem}

As in [1] it will be assumed that $T_{o}$ is chosen to be small enough such that the parameters of interest remain approximately constant over the update interval: $\boldsymbol{\xi}(k) \approx \boldsymbol{\xi}(t), t \in$ $\left[k T,(k+1) T_{o}\right)$. Thus, the correlation matrix $\mathbf{R}=E\left[\mathbf{z z}^{H}\right]$ remains roughly constant over the update interval and can be estimated by: $\widehat{\mathbf{R}}(k)=\frac{1}{B} \sum_{b=1}^{B} \mathbf{z}_{k}(b) \mathbf{z}_{k}^{H}(b)$ where the estimated correlation matrix and the snapshot vectors are shown with the track update index, $k$.

Now, the problem addressed in this paper is how to use the received data at frequency $\omega$ and the dynamical models introduced above to track the source DOA's and array shape over time such that correct source-to-angle association is maintained. That is, for each $k \in Z_{+},\left\{\hat{\theta}_{n}(k)\right\}_{n=1}^{N}$, estimates of the source DOA's are to be formed so that $\hat{\theta}_{n}(k)$ always corresponds to an estimate of the DOA of the $n$th source in particular.

\section{MAXIMUM LIKELIHOOD ESTIMATION}

This section describes how the deterministic likelihood function for the problem of joint DOA/array shape estimation is used to provide the initial parameter estimates. If the source waveforms and the sensor positions are modeled as deterministic unknowns, it is not difficult to show that their deterministic MLE's are given as [6]:

$$
\begin{aligned}
\widehat{\boldsymbol{\xi}}_{M L} & =\arg \min _{\boldsymbol{\xi}}\{L(\boldsymbol{\xi})\} \\
L(\boldsymbol{\xi}) & =\operatorname{tr}\left(\mathbf{P}_{\mathbf{A}}^{\perp}(\boldsymbol{\xi}) \widehat{\mathbf{R}}\right) \\
\mathbf{P}_{\mathbf{A}}^{\perp}(\boldsymbol{\xi}) & =\mathbf{I}-\mathbf{A} \mathbf{A}^{\#}, \quad \mathbf{A}^{\#}=\left(\mathbf{A}^{H} \mathbf{A}\right)^{-1} \mathbf{A}^{H} \\
\boldsymbol{\xi} & =\left[\theta_{1}, \cdots, \theta_{N}, y_{3}, \cdots, y_{M}\right]^{T}
\end{aligned}
$$

where the parameter vector will contain the $N$ source DOA's followed by $M-2 y$-axis sensor displacements. $\operatorname{tr}(\cdot)$ denotes the matrix trace operation, and $\mathbf{P}_{\mathbf{A}}^{\perp}(\xi)$ and $\mathbf{A}^{\#}$ are, respectively, the orthogonal complement projector and the pseudoinverse associated with the steering matrix, $\mathbf{A}$. To simplify notation, the dependence on the angle update time index $k$ and the parameter vector, $\xi$, has been and will be dropped when possible. Also note that the number of sources, $N$ is assumed known and constant.

In the context of tracking, MLE's of the parameters can be obtained by iteratively minimizing the likelihood cost function of (8). In particular, given $\xi_{0}$, a parameter vector known to be "close" to the MLE parameter vector, the MLE's can be obtained by an iterative Gauss-Newton procedure as in [8]. In practice, $\boldsymbol{\xi}_{0}$, can be obtained readily from the parameter estimate of the previous iteration or some function thereof. (The details are provided in the next section.) Since this will often be a good initial guess at the maximum likelihood parameter vector, $\widehat{\xi}_{M L}$, a low number of Gauss-Newton iterations will normally be required. It is $\widehat{\xi}_{M L}$ that will be applied to the Kalman filtering procedure described in the next section. 


\section{TRACKING ALGORITHM}

The measurement equation corresponding to the dynamic model (5) describing the DOA trajectory of the $n$th source at time $k$ is:

$$
g_{\theta_{n}}(k)=\mathbf{h}_{\theta}^{T} \mathbf{x}_{\theta_{n}}(k)+v_{\theta_{n}}(k), \quad \mathbf{h}_{\theta}^{T}=\left[\begin{array}{ll}
1 & 0
\end{array}\right]^{T}
$$

Since the measurement is taken as the MLE for the DOA of the $n$th source, $g_{\theta_{n}}(k)=\left[\widehat{\xi}_{M L}(k)\right]_{n}=\hat{\theta}_{M L_{n}}$, as computed in (7), $\mathbf{h}_{\theta}^{T} \mathbf{x}_{\theta_{n}}(k)$ and $v_{\theta_{n}}(k)$ are respectively identified as the true $D O A, \theta_{n}(k)$, and the $M L E$ error. As in [1], if the measurement noises (i.e., estimation errors) of the $N$ DOA estimates are assumed to be zero mean Gaussian and mutually uncorrelated, the problem can be decoupled into a bank of scalar Kalman filters (one for each source) with significant savings in computation.

More specifically the Kalman prediction is formed as:

$$
\hat{\mathbf{x}}_{\theta_{n}}(k \mid k-1)=\mathbf{F}_{\theta} \hat{\mathbf{x}}_{\theta_{n}}(k-1 \mid k-1),
$$

where $\hat{\mathbf{x}}_{\theta_{n}}(k \mid k-1)$ and $\hat{\mathbf{x}}_{\theta_{n}}(k-1 \mid k-1)$ denote, respectively, the predicted state vector for the $n$th source DOA at time $k$ and the filtered state vector at time $k-1$. Next, the MLE's of all the parameters are formed by via a Gauss-Newton iteration initialized at $\boldsymbol{\xi}_{0}=\widehat{\boldsymbol{\xi}}(k \mid k-1)$, the parameter vector obtained from the Kalman predictions, and is used such that the state update equation can be written as:

$$
\begin{aligned}
\hat{\mathbf{x}}_{\theta_{n}}(k \mid k)=\hat{\mathbf{x}}_{\theta_{n}}(k \mid k-1)+\mathbf{k}_{\theta_{n}}(k) \\
\cdot\left[\hat{\theta}_{M L_{n}}(k)-\hat{\theta}_{n}(k \mid k-1)\right]
\end{aligned}
$$

where $\hat{\mathbf{x}}_{\theta_{n}}(k \mid k)$ and $\mathbf{k}_{\theta_{n}}(k)$ are, respectively, the filtered state vector and the Kalman gain vector for the $n$th source DOA at time $k$. It is noted that the MLE of the DOA of the $n$th source minus the predicted angle serves as the innovation sequence of the associated Kalman filter. The final angle estimate for the $n$th source at time $k$ is given by the first element of $\hat{\mathbf{x}}_{\theta_{n}}(k \mid k)$.

The Kalman gain is updated by the following set of equations:

$$
\begin{aligned}
\mathbf{k}_{\theta_{n}}(k)= & \frac{\boldsymbol{\Sigma}_{\theta_{n}}(k \mid k-1) \mathbf{h}_{\theta}}{\mathbf{h}_{\theta}^{T} \boldsymbol{\Sigma}_{\theta_{n}}(k \mid k-1) \mathbf{h}_{\theta}+r_{\theta_{n}}(k)} \\
\boldsymbol{\Sigma}_{\theta_{n}}(k \mid k)= & \boldsymbol{\Sigma}_{\theta_{n}}(k \mid k-1) \\
& -\mathbf{k}_{\theta_{n}}(k) \mathbf{h}_{\theta}^{T} \mathbf{\Sigma}_{\theta_{n}}(k \mid k-1) \\
\boldsymbol{\Sigma}_{\theta_{n}}(k+1 \mid k)= & \mathbf{F}_{\theta} \mathbf{\Sigma}_{\theta_{n}}(k \mid k) \mathbf{F}_{\theta}^{T}+\mathbf{Q}_{\theta_{n}}(k) .
\end{aligned}
$$

$r_{\theta_{n}}(k)$, is effectively a gauge of the quality of the MLE for the $n$th source DOA produced by (7) at time $k$. In practice, control of $r_{\theta_{n}}(k)$ has been observed to be important for proper performance of the tracker-especially when sources cross in angle. It is reasonable and convenient to set the measurement noise variance as the $n$th element on the diagonal of the Cramer-Rao Bound (CRB) for this problem (assuming Gaussian signals):

$$
\begin{aligned}
r_{\theta_{n}}(k) & =\mathbf{C R B}_{n n} \\
\mathbf{C R B} & =\mathbf{J}^{-1} \\
{[\mathrm{~J}]_{m n} } & =E \operatorname{tr}\left(\mathbf{R}^{-1} \frac{\partial \mathbf{R}}{\partial \xi_{m}} \mathbf{R}^{-1} \frac{\partial \mathbf{R}}{\partial \xi_{n}}\right) .
\end{aligned}
$$

It should be emphasized that the CRB for the DOA's increases dramatically for sources that are closely spaced in angle relative to the resolution of the array. In such cases, the corresponding increase in $r_{\theta_{n}}(k)$ has the effect of greatly decreasing the Kalman gains of the associated Kalman filters. This is intuitively desirable since the Kalman filters tracking the crossing sources should depend much more on the constant velocity model via (11) than the (extremely noisy) measurements produced by (7).

The Kalman filtering equations for the discrete spatiotemporally correlated sensor motion model (6) are analogous and therefore not shown. It is worth noting that since, unlike the measurement noise variance for the source DOA's (which can easily fluctuate several order of magnitude), that of the $y$-axis sensor displacements remains relatively constant. Thus, additional savings in computation can be obtained by setting the sensor displacement measurement noises to reasonable constant values, $\left\{r_{y_{m}}\right\}_{m=i}^{M}$, and calculating the Kalman gain update equations in advance off-line.

\section{RESULTS}

Consider the case of $N=3$ unit power (OdB) narrowband sources centered at $f=100 \mathrm{~Hz}$ impinging on a towed ULA of $M=20$ sensors with nominal half-wavelength $(\lambda / 2=7.5 m)$ inter-element spacing along the $x$-axis. The array length is $(M-1) \lambda / 2=142.5 \mathrm{~m}$. The sources have initial DOA's of $\theta_{1}(0)=-20^{\circ}, \theta_{2}(0)=-10^{\circ}$, and $\theta_{3}(0)=$ $25^{\circ}$. Source three is a fully coherent multipath reflection of source two. Additive sensor noise of variance $-20 \mathrm{~dB}$ is also present. Six minutes of the source trajectories are shown in Fig. 1. Sinusoidal motion of amplitude $20 \mathrm{~m}$ and frequency $2 \mathrm{mHz}$ at the tow point propagates down the array according to the water pulley solution of the Paidoussis equation. The disturbance is assumed to propagate undamped along the array $(\alpha=1)$ at the tow speed, $2.5 \mathrm{~m} / \mathrm{sec}$. The tracker is updated once every three seconds (i.e., $T=3$ ) implying that $\rho=1$.

If the array shape tracker of [4], [5] uses a water pulley sensor motion model which nearly perfectly describes the actual sensor motion, highly accurate sensor positions estimates can be obtained and then used in a DOA only tracker. However, in practice, modeling errors may arise due to ocean induced array motion or the discretization of the Paidoussis equation [4] as well as imprecisely modeled damping along the array, inaccuracies in the assumed tow speed or the assumed speed with which the tow point induced motion propagates along the array. Assume that the array is outfitted with depth sensors at hydrophone sensor positions $m \in\{1,2,11\}$ which yield estimates of the array position at these points which are unbiased and of variance, $10^{-4} \mathrm{~m}^{2}$. Let us consider the same scenario again with assumed tow speed $2.5 \mathrm{~m} / \mathrm{sec}$ and assumed damping $\alpha=1$ but with true tow speed $2.48 \mathrm{~m} / \mathrm{sec}$ and true damping 0.99. Fig. 2 indicates that these small errors (each one percent of the true values) produce significant MSE which propagates along the array. Moreover, applying these sensor position estimates to the DOA only tracker has been seen to cause this tracker to fail completely.

Next, the joint DOA-array shape tracker performance is considered. For the same scenario lower MSE in sensor position at each sensor is indicated in Fig. 3. The bias 
and variance of the source DOA's as a function of time are shown in Figs. 4 and 5, respectively. The graphs are based on averaging two hundred independent realizations. $B=20$ frequency domain snapshots at $f=100 \mathrm{~Hz}$ are available per tracker iteration. As opposed to the decoupled trackers, here successful joint-DOA array shape tracking was obtained in the presence of array motion modeling errors and without relying on the depth sensor at $m=11$. Transient effects are observed approximately until $t=50$ to $t=100 \mathrm{sec}$ (i.e., for about 17 to 34 tracker iterations). The slight bias seen when sources cross is believed to be due the Kalman filters' almost exclusive reliance on the constant angular velocity model approximation during and near source crossings. Simulations (not shown) were also conducted for high noise and widely disparate source power scenarios. It was found that, as expected, performance worsened as SNR decreased.

\section{CONCLUSION}

A new joint DOA/array shape tracking algorithm for multiple moving targets in the presence of sensor motion has been presented. The technique applies the output of the MLE to a bank of post-processing Kalman filters effectively reducing the tracker's dependence on depth sensors and compasses and providing robust performance in the presence of sensor motion modeling errors. Extensions to near-field and/or wideband sources are straightforward.

\section{REFERENCES}

[1] C.R. Rao, C.R. Sastry, and B. Zhou. "Tracking the direction of arrival of multiple moving targets". IEEE Transactions on Signal Processing, vol. 42: pp. 11331144, May 1994.

[2] Y.Rockah and P.M. Schultheiss. "Array shape calibration using sources in unknown locations-Part I: Far-field sources". IEEE Transactions on Acoustics, Speech, and Signal Processing, vol. 35: pp. 286-299, March 1987.

[3] B.E. Howard and J.M. Syck. "Calculation of the shape of a towed underwater acoustic array". IEEE Journal of Oceanic Engineering, vol. 17: pp. 193-203, April 1992 .

[4] D.A. Gray, B.D.O. Anderson, and R.R. Bitmead. "Towed array shape estimation using Kalman filterstheoretical models". IEEE Journal of Oceanic Engineering, vol. 18: pp. 543-556, October 1993

[5] J.L. Riley and D.A. Gray. "Towed array shape estimation using Kalman filters-experimental investigations". IEEE Journal of Oceanic Engineering, vol. 18: pp. 572581 , October 1993

[6] A.J. Weiss and B. Friedlander. "Array shape calibration using sources in unknown locations-a maximum likelihood approach". IEEE Transactions on Acoustics, Speech, and Signal Processing, vol. 37: pp. 1958-1966, December 1989

[7] P.M. Schultheiss and H. Messer. "Optimal and suboptimal broad-band source location estimation". IEEE Transactions on Signal Processing, vol. 41: pp. 27522763, September 1993.

[8] M. Viberg, B. Ottersten, and T. Kailath. "Detection and estimation in sensor arrays using weighted subspace fitting". IEEE Transactions on Signal Process. ing, vol. 39: pp. 2436-2449, November 1991.

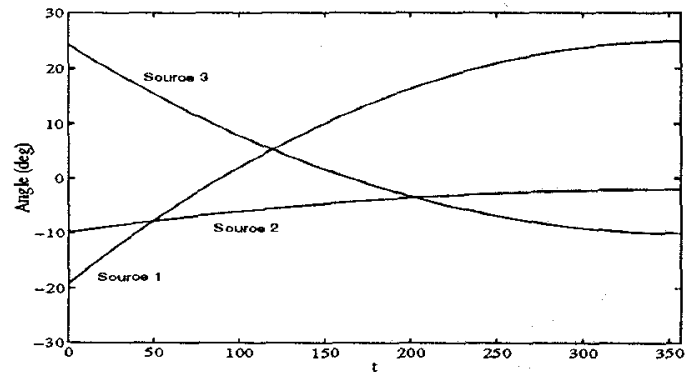

Figure 1: Source DOA trajectories (degrees).

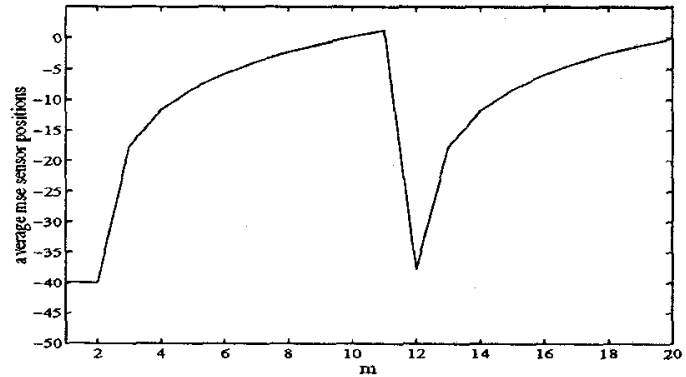

Figure 2: Decoupled Tracker-Sensor position MSE $\left(\mathrm{m}^{2}\right)$.

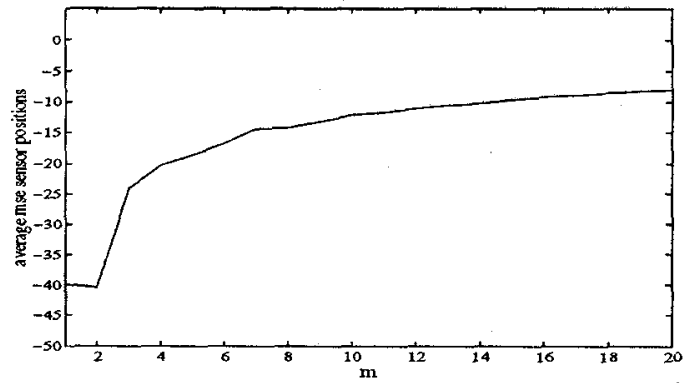

Figure 3: Joint Tracker-Sensor position MSE $\left(m^{2}\right)$.
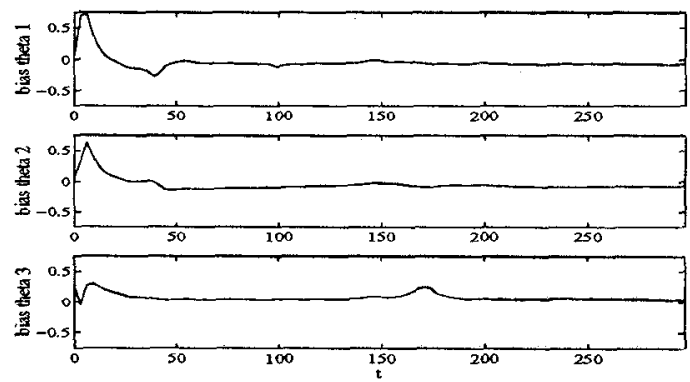

Figure 4: Joint Tracker-DOA bias (degrees).
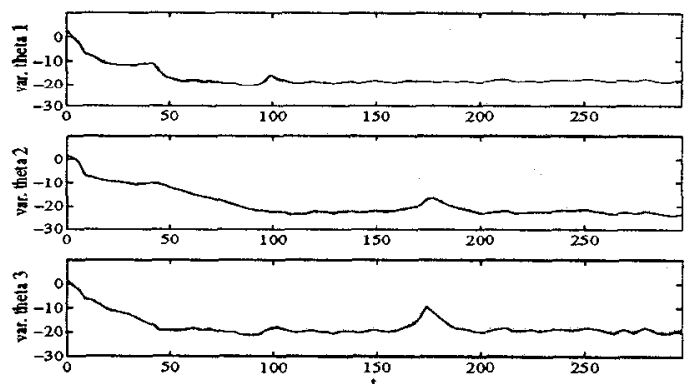

Figure 5: Joint Tracker-DOA variance (degrees sqrd). 